Aparecida de Cássia Rabetti

Sérgio Fernando Torres de Freitas

\section{Avaliação das ações em hipertensão arterial sistêmica na atenção básica}

\section{Evaluation of actions concerning systemic arterial hypertension in primary healthcare}

RESUMO

OBJETIVO: Avaliar a eficiência da Estratégia Saúde da Família nas ações relacionadas à hipertensão.

MÉTODOS: Estudo avaliativo, transversal quantitativo, com base em dados secundários de 66 municípios catarinenses de pequeno porte, com cobertura potencial máxima de 100\% pela Estratégia Saúde da Família em 2007. Foram avaliados indicadores de insumos, produtos e resultados. A eficiência da produção de serviços e da produção de resultados dos municípios foi comparada por meio de análise envoltória de dados.

RESULTADOS: Os municípios foram mais eficientes na produção de serviços $(37,8 \%)$ do que na produção de resultados (16,6\%). Quarenta e um municípios $(62,2 \%)$ foram ineficientes nos serviços: cadastro no Sistema de Informação sobre Hipertensão e Diabetes, atendimento individual e visita domiciliar para usuários com hipertensão, e $55(83,3 \%)$ foram ineficientes na produção de impacto contra hipertensão.

CONCLUSÕES: O modelo de avaliação utilizado mostrou-se capaz de medir a eficiência na atenção primária de saúde, ao avaliar a produtividade de serviços e de resultados.

DESCRITORES: Hipertensão, prevenção \& controle. Programa Saúde da Família. Avaliação de Serviços de Saúde. Atenção Primária à Saúde. Estudos Transversais.
Núcleo de Extensão e Pesquisa em Avaliação em Saúde. Departamento de Saúde Pública. Universidade Federal de Santa Catarina. Florianópolis, SC, Brasil

Correspondência | Correspondence: Aparecida de Cássia Rabetti

Núcleo de Extensão e Pesquisa em Avaliação em Saúde - Nepas

Campus Universitário Trindade

88040-970 Florianópolis, SC, Brasil

E-mail: cassia_rabetti@hotmail.com

Recebido: 24/3/2010

Aprovado: 25/8/2010

Artigo disponível em português e inglês em: www.scielo.br/rsp 


\section{ABSTRACT}

OBJECTIVE: To evaluate the efficiency of the Family Health Strategy in actions related to hypertension.

METHODS: Evaluative, cross-sectional quantitative research based on secondary data of 66 small municipalities located in the state of Santa Catarina, Southern Brazil, with maximum potential coverage of $100 \%$ by the Family Health Strategy in 2007. Input indicators, products and results were evaluated. The municipalities' efficiency of services production and results production was compared through data envelopment analysis.

RESULTS: The municipalities were more efficient in services production $(37.8 \%)$ than in results production (16.6\%). Forty-one municipalities $(62.2 \%)$ were inefficient in the services: enrolment in the Hypertension and Diabetes Information System, individual assistance and home visit for hypertensive users, and $55(83.3 \%)$ were inefficient in the production of impact against hypertension.

CONCLUSIONS: The evaluation model used in this study proved to be capable of measuring efficiency in primary healthcare by evaluating the productivity of services and results.

\section{DESCRIPTORS: Hypertension, prevention \& control. Family Health Program. Health Services Evaluation. Primary Health Care. Cross- Sectional Studies.}

\section{INTRODUÇÃO}

Os gastos públicos com saúde são crescentes e relacionados a fatores como envelhecimento da população, novas tecnologias em saúde, melhoria nos níveis de renda, consolidação do estado de bem-estar e da universalização da cobertura sanitária. ${ }^{a}$ Nessas circunstâncias, o uso de instrumentos metodológicos econômicos em saúde é justificado pelo critério de escassez e pela dificuldade de alocação de recursos. ${ }^{16}$

A análise envoltória de dados (data envelopment analysis - DEA), elaborada nos anos 1980, mede a eficiência produtiva na área social. Foi desenvolvida para avaliação de programas públicos, em que não predomine ou não seja considerado somente o aspecto financeiro das organizações. Essa ferramenta é aplicada em estudos de produtividade e eficiência técnica de unidades produtivas que empregam múltiplos insumos para gerar múltiplos produtos e permite identificar as melhores práticas por meio de fronteiras empíricas de programação linear. $^{6}$

Desde sua implantação, o Sistema Único de Saúde (SUS) lida com insuficiência de recursos, dado cenário adverso do ponto de vista econômico à época de sua criação. O Programa de Saúde da Família surgiu sob a crítica inicial de ser um programa com características restritivas de atenção. Entretanto, sua expansão veloz nos últimos anos e sua importância a transformaram numa estratégia de conversão do modelo de atenção básica. O controle e diagnóstico da hipertensão tem sido atribuição da Saúde da Família, tem caráter de ação prioritária na saúde do adulto em sua fase inicial e é ação estratégica de atuação após o Pacto em Defesa da Vida, de $2005 .{ }^{1}$ A hipertensão arterial sistêmica (HAS) é uma doença crônica não-transmissível de alta prevalência, cujo diagnóstico e controle são imprescindíveis no manejo de graves doenças, como insuficiência cardíaca congestiva, doenças cerebrovasculares, infarto agudo do miocárdio, nefropatia hipertensiva, insuficiência vascular periférica e retinopatia hipertensiva.

A avaliação da eficiência dos serviços relacionados à HAS com a identificação de pontos fortes de atuação de municípios de referência poderia representar importante ferramenta de gestão e planejamento. Isso porque possibilitaria a melhoria da atenção, ao identificar municípios e ações com impacto eficiente, e assim subsidiaria informações sobre como produzir serviços e resultados com maior eficiência. O objetivo do presente estudo foi avaliar a eficiência da Estratégia Saúde da Família (ESF) nas ações relacionadas à hipertensão.

a Ministério da Saúde. Secretaria-Executiva. Área de Economia da Saúde e Desenvolvimento. Avaliação econômica em saúde: desafios para gestão no Sistema Único de Saúde. Brasília (DF); 2008. 


\section{MÉTODOS}

Foram selecionados 66 municípios de pequeno porte de Santa Catarina, cujo modelo de Atenção Primária à Saúde (APS) era a ESF para toda a população.

Informações sobre os municípios catarinenses referentes a 2007 foram coletadas nos bancos de dados do Sistema de Informação da Atenção Básica (Siab), Sistema de Informação de Internação Hospitalar, Cadastro Nacional de Estabelecimentos de Saúde, base populacional do Instituto Brasileiro de Geografia e Estatística, Sistema de Informação Orçamentária Pública em Saúde e Sistema de Informação sobre Hipertensão e Diabete (Sishiperdia).

Um modelo avaliativo foi construído conforme preconizado por Rabetti (2009). ${ }^{\mathrm{b}}$ Os dados foram organizados em insumos e produtos relacionados à HAS na atenção básica. Recursos financeiros, materiais e força de trabalho foram considerados como insumos para a produção de serviços, e ações para controle e diagnóstico da HAS, como produtos. Os serviços gerados foram transformados em insumos e tiveram como produto o controle dos agravos imediatos e mediatos decorrentes da HAS (Figura 1).

Os insumos foram: recurso financeiro (total aplicado pelo município para custeio da atenção básica); recurso material (horas semanais de ocupação de consultórios destinados a atenção básica); e força de trabalho (jornada semanal média das equipes de Saúde da Família por mês).

Vinculação (número de indivíduos com HAS cadastrados), atendimento individual (número de atendimentos à HAS realizadas pela $\mathrm{SF}$ ) e visita domiciliar (número de visitas realizadas por agentes comunitários de saúde aos usuários com HAS) foram os serviços considerados.

Os serviços relacionados à HAS desempenhados pela ESF foram classificados como insumos na etapa de produção de resultados. O número de serviços observados foi corrigido para número de serviços projetados para eficiência pela ferramenta DEA, a fim de manter a relação com os insumos iniciais.

Foi criado um indicador de resultado ou taxa que representasse a proteção aos desfechos cardiovasculares $(\mathrm{CV})$. A essa taxa denominou-se taxa de internações potencialmente evitadas pela atenção primária relacionadas à HAS, calculada pela fórmula: taxa $=$ [(população entre 20 e 65 anos - número de internações

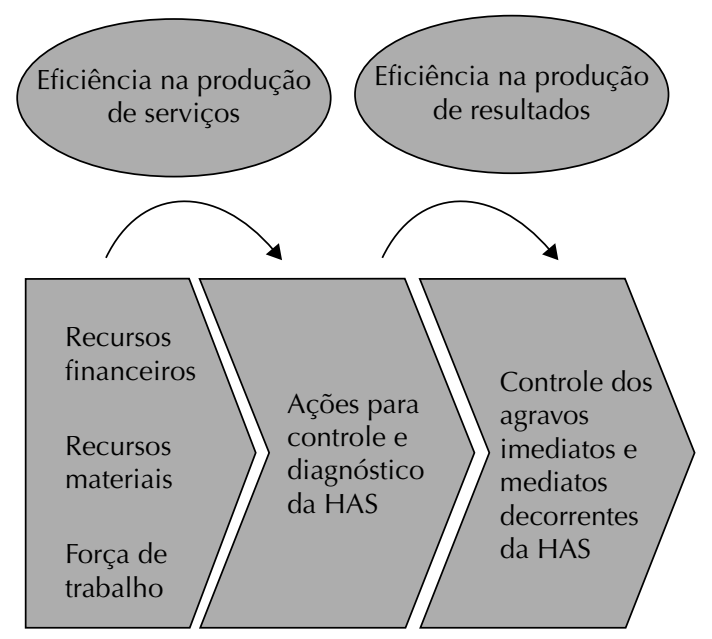

Figura 1. Modelo teórico da eficiência na produção de serviços e resultados das ações relacionadas à hipertensão arterial sistêmica (HAS) na atenção básica. Santa Catarina, 2007

por HAS, Insuficiência Cardíaca Congestiva e Doenças Cerebrovasculares em indivíduos entre 20 e 65 anos) : população entre 20 e 65 anos] $* 10.000$.

A DEA foi empregada para construir fronteiras empíricas de eficiência produtiva, isto é, agrupamento das melhores produtividades observadas, formando um conjunto de unidades de produtividade máxima, e nenhuma unidade produtiva está acima desse limite. Uma de suas maiores vantagens é a eficiência identificada, que é real e não calculada como uma combinação teórica do melhor que poderia ser feito em condições ideais. $^{c}$

Nos métodos DEA, as unidades de análise são avaliadas conforme a utilização de insumos para produção de determinada quantidade de produtos. Pesos são atribuídos para cada insumo e para cada produto visando a uma relação mais eficiente.

DEA foi escolhida pela possibilidade de análises complexas para múltiplos insumos e múltiplos produtos; por não requerer existência predeterminada de um modelo matemático que relacione insumos e produtos (a única hipótese mantida é que a soma ponderada de insumos e produtos de qualquer município resulta em um "município virtual" de tecnologia factível); por comparar unidades diretamente com seu par ou com uma combinação de pares; e porque os insumos e produtos podem se expressar em unidades diferentes (por exemplo: número de equipes investidas e valores investidos em reais). ${ }^{8}$

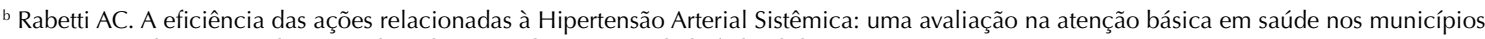
catarinenses [dissertação de mestrado]. Florianópolis: Universidade federal de Santa Catarina; 2009.

${ }^{c}$ Calvo MCM. Análise da eficiência produtiva de hospitais públicos e privados no Sistema Único de Saúde (SUS). In: Piola SF, Jorge EA. Prêmio em economia da saúde: $1^{\circ}$ prêmio nacional, 2004: coletânea premiada. Brasília (DF): Instituto de Pesquisa Econômica Aplicada; 2005.
} 
As principais limitações na DEA são os erros de medida e a presença de outliers, que interferem na formação da fronteira. Por isso, recomenda-se a checagem da presença de observações influentes e de outliers. ${ }^{8}$

Restrições foram estabelecidas para controlar a heterogeneidade dos municípios e para que fossem estruturalmente comparáveis: mesmo porte $(\leq 10$ mil habitantes) e mesma cobertura de ESF $(100 \%$ de cobertura potencial máxima). Para controle dos outliers, foram excluídos 37 municípios (35,9\%) por ausência de dados ou por erros de medidas não passíveis de correção. DEA foi aplicada nas duas etapas produtivas por meio do programa Ideas ${ }^{\circledR} .{ }^{c} \mathrm{O}$ modelo de escalas variáveis foi utilizado, sob a hipótese de que a variação no porte do município poderia interferir na escala de produção de serviços e resultados. DEA foi orientada para produtos, com o arranjo de insumos investidos, que buscou a melhor produtividade e aumentou o número de produtos.

As produtividades máximas observadas formaram uma fronteira empírica de eficiência entre os municípios catarinenses de pequeno porte. A produtividade máxima observada assumiu escore igual a 1 e foi classificada como eficiente; escore superior a 1 foi categorizado como ineficiente.

Por meio da DEA foram calculadas metas eficientes de produtividade para as unidades ineficientes e a ineficiência para cada produto foi avaliada.

\section{RESULTADOS}

Dos 66 municípios, 25 (37,8\%) foram eficientes na produção de serviços relacionados à HAS e 41 , ineficientes $(62,2 \%)$.

Tabela 1. Municípios de referência benchmarks para produção de serviços de atenção à hipertensão arterial sistêmica efetuados pela Estratégia Saúde da Família, seus insumos e produtos. Santa Catarina, 2007.

\begin{tabular}{|c|c|c|c|c|c|c|c|}
\hline \multirow[b]{2}{*}{ Municípios } & \multirow[b]{2}{*}{$\begin{array}{l}\text { Número } \\
\text { de } \\
\text { vezes de } \\
\text { referência }\end{array}$} & \multicolumn{3}{|c|}{ Insumos consumidos } & \multicolumn{3}{|c|}{ Serviços produzidos } \\
\hline & & $\begin{array}{l}\text { Investimento } \\
\text { (milhões de } \\
\text { reais) }\end{array}$ & $\begin{array}{c}\text { Jornada de } \\
\text { trabalho de } \\
\text { Equipes de Saúde } \\
\text { da Família (h/sem) }\end{array}$ & $\begin{array}{l}\text { Utilização } \\
\text { de } \\
\text { consultórios } \\
\text { (h/sem) }\end{array}$ & $\begin{array}{l}\text { Vinculação } \\
\text { do usuário }\end{array}$ & $\begin{array}{l}\text { Atendimento } \\
\text { individual }\end{array}$ & $\begin{array}{c}\text { Visitas } \\
\text { domiciliares }\end{array}$ \\
\hline Petrolândia & 25 & $1.178,40$ & 80 & 40 & 362 & 570 & 10.103 \\
\hline Serra Alta & 15 & $1.300,00$ & 40 & 40 & 491 & 547 & 4.737 \\
\hline Descanso & 14 & $1.968,25$ & 113 & 120 & 821 & 4.834 & 13.967 \\
\hline Bandeirante & 11 & $1.365,64$ & 37 & 40 & 280 & 225 & 4.988 \\
\hline Zortéa & 11 & 271,55 & 40 & 40 & 123 & 3.283 & 3.076 \\
\hline Tunápolis & 10 & $1.214,14$ & 80 & 40 & 689 & 3.599 & 6.878 \\
\hline Major Gercino & 9 & $1.037,98$ & 37 & 120 & 133 & 5.254 & 4.310 \\
\hline Abdon Batista & 8 & $1.001,41$ & 33 & 40 & 252 & 275 & 3.978 \\
\hline Jaborá & 6 & 605,83 & 80 & 120 & 648 & 1.147 & 6.779 \\
\hline Novo Horizonte & 6 & 559,77 & 40 & 40 & 283 & 1.820 & 3.006 \\
\hline Quilombo & 6 & $3.568,30$ & 160 & 240 & 667 & 17.759 & 5.684 \\
\hline Cunhataí & 5 & $1.035,30$ & 27 & 40 & 101 & 3.466 & 3.064 \\
\hline $\begin{array}{l}\text { São Pedro de } \\
\text { Alcântara }\end{array}$ & 5 & $1.392,86$ & 63 & 240 & 448 & 5.502 & 6.684 \\
\hline Trombudo Central & 5 & $1.638,81$ & 80 & 40 & 227 & 7.799 & 7.825 \\
\hline Vargem Bonita & 5 & $2.197,73$ & 80 & 240 & 963 & 2.593 & 7.500 \\
\hline Águas Frias & 3 & $1.154,12$ & 40 & 40 & 386 & 2.136 & 3.900 \\
\hline Bom Retiro & 3 & $1.685,64$ & 120 & 240 & 1.072 & 670 & 11.592 \\
\hline São João do Oeste & 3 & $1.518,50$ & 80 & 80 & 732 & 1.723 & 8.789 \\
\hline Erval Velho & 2 & $1.476,89$ & 77 & 80 & 764 & 351 & 6.921 \\
\hline Rio dos Cedros & 2 & $2.438,33$ & 157 & 160 & 324 & 7.147 & 13.771 \\
\hline Alfredo Wagner & 1 & $2.071,48$ & 120 & 160 & 871 & 4.875 & 11.513 \\
\hline Imbuia & 1 & 494,63 & 80 & 80 & 250 & 1.272 & 6.194 \\
\hline Jupiá & 1 & 888,40 & 33 & 40 & 224 & 1.834 & 2.675 \\
\hline Luzerna & 1 & $1.558,99$ & 80 & 120 & 778 & 2.175 & 8.618 \\
\hline Nova Itaberaba & 1 & $1.647,62$ & 80 & 80 & 98 & 9.174 & 5.779 \\
\hline
\end{tabular}




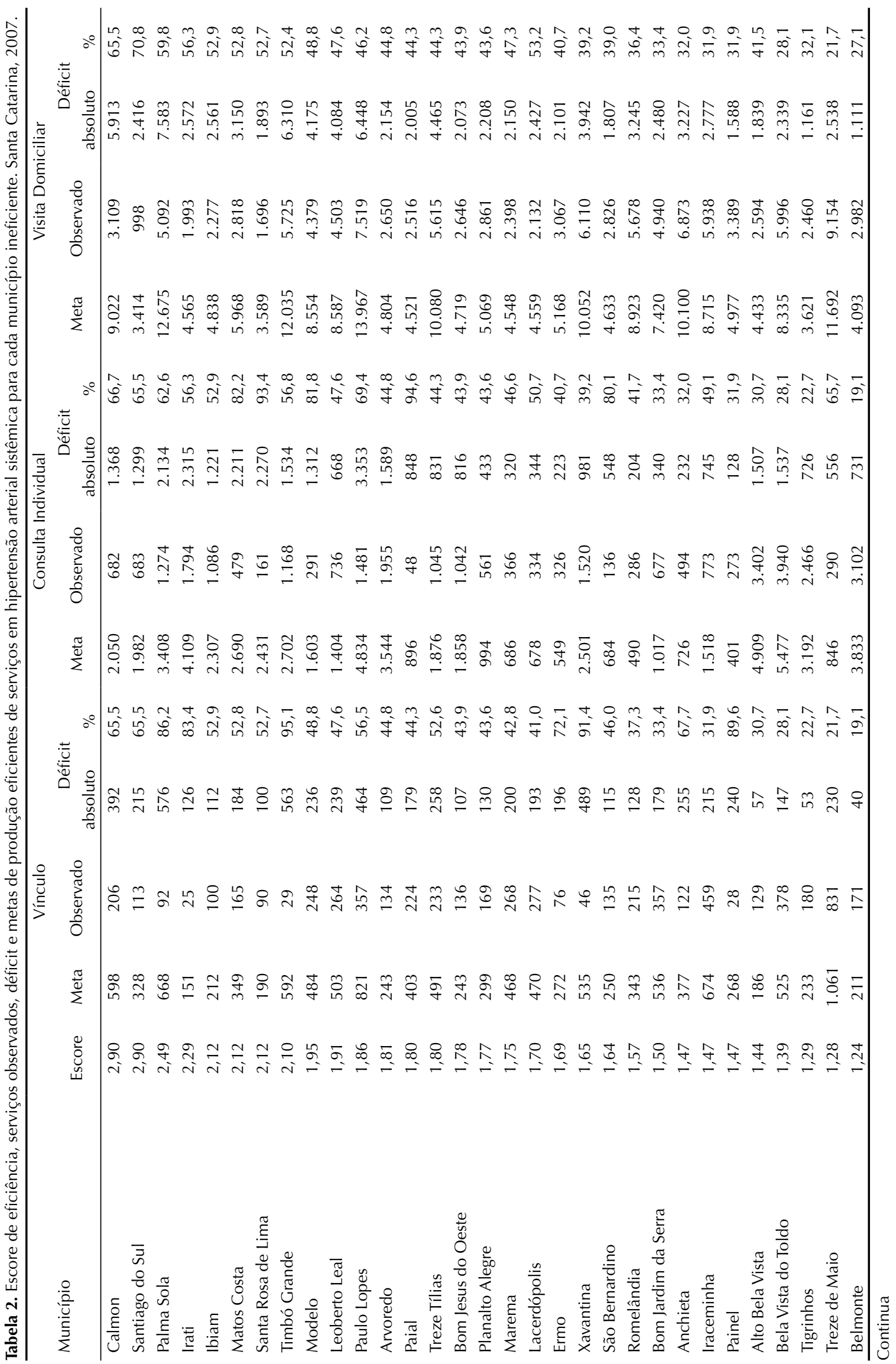




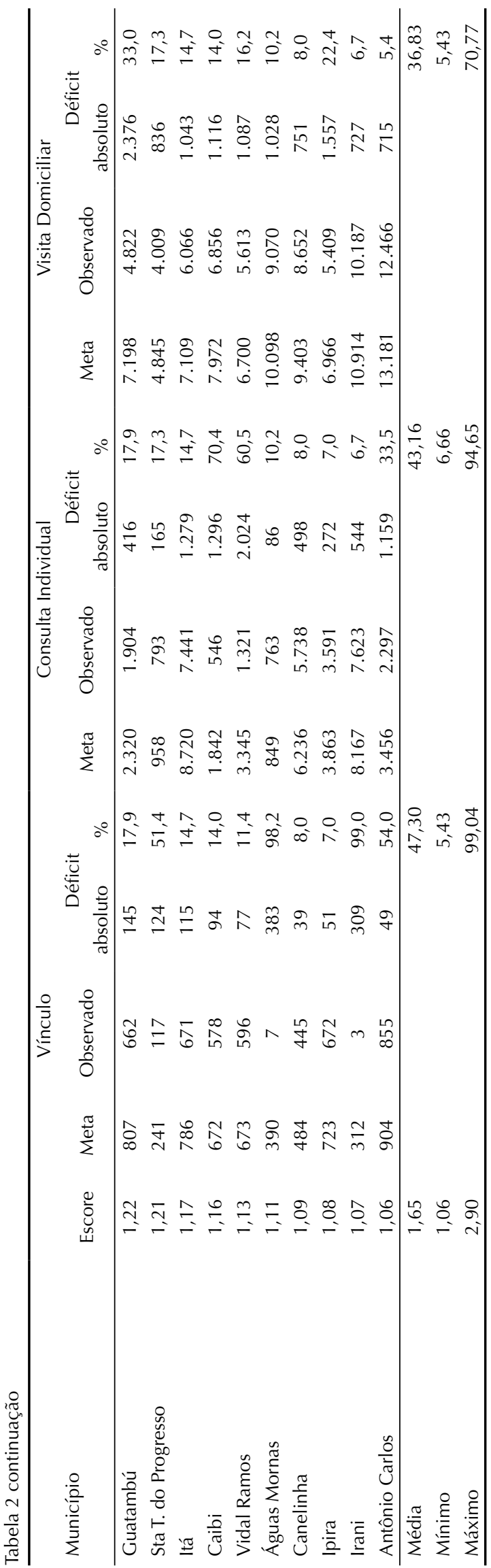

Os escores dos municípios ineficientes variaram de 1,06 a 2,9 (produtividades 0,6 a 1,9 menores que a produtividade máxima observada). Os municípios eficientes com arranjos de insumos semelhantes conseguiram produzir mais serviços e tornaram-se referência para os demais.

Municípios considerados ineficientes tiveram mais de um município como referência. A Tabela 1 mostra os benchmarks em serviços e as características de suas práticas. $\mathrm{O}$ total de referências foi maior que o de observações, pois houve municípios com mais de uma referência.

Cinco municípios foram referência somente para si, i.e., os demais não conseguiram projeções para suas práticas a partir de seus arranjos.

Dentre os municípios, 41 foram ineficientes para a produção dos três tipos de serviços: vinculação do usuário, atendimento individual e visita domiciliar (Tabela 2).

A maior ineficiência foi na vinculação de usuários medida pelo cadastro de usuários com HAS no Sishiperdia, com média de $47,3 \%$ no estado. O número de cadastrados foi incompatível com as demais atividades observadas em alguns municípios (Tabela 2). O município Irani, o mais ineficiente, tinha três usuários cadastrados, quando deveria ter 312 ; no entanto, ofereceu 7.623 consultas individuais para essa atividade e pouco mais de 10 mil visitas domiciliares, o que sugere deficiência específica no cadastro de usuários.

Os municípios precisavam expandir a produção em $43,16 \%$ em média para atendimento individual. $\mathrm{O}$ município Paial teve a maior ineficiência em atendimento individual, com oferta de 48 consultas para usuários com HAS em um ano. Para se tornar eficiente, sua meta produtiva seria de 896 consultas/ano, o que contabilizaria incremento de $94,7 \%$ nesse produto. A ineficiência foi menor nos outros serviços (cerca de 40\%) para esse município.

O menor grau de ineficiência foi observado para visitas domiciliares. Santiago do Sul foi o mais ineficiente em visita domiciliar, com 998 visitas e meta para alcançar a eficiência de 3.414. Esse município apresentou ineficiência distribuída eqüitativamente entre os três serviços.

Considerando-se a produção de resultados, 11 municípios $(16,7 \%)$ foram eficientes quanto aos cuidados com HAS na atenção básica; os demais 55 (83,3\%) foram ineficientes.

A fronteira de eficiência na produção de resultados foi constituída por menos municípios que a fronteira de serviços. Os escores dos municípios ineficientes foram próximos a 1, o que sugere que sua produtividade máxima quase foi alcançada. A variação dos escores ineficientes foi de 1,000014 a 1,006635 . 
Dos 11 municípios eficientes na produção de resultados, nove $(81,9 \%)$ tornaram-se referência para outros ineficientes. Dois $(18,1 \%)$, apesar de eficientes, não tinham municípios que pudessem ser projetados para suas práticas (Tabela 3). O município Jaborá foi o que mais serviu de referência (36 municípios podem alcançar seus resultados sem modificar seus insumos). Esse benchmark apresentou duas internações pelas causas selecionadas. Assim, 9.991 a cada 10 mil pessoas da população suscetível foram protegidas dos desfechos $\mathrm{CV}$, o que indica que os serviços desenvolvidos pela ESF protegeram essa parcela da população. Essa taxa aplicada à população suscetível mostrou que houve a proteção máxima que o serviço poderia obter.

Nos municípios ineficientes, em média, 18:10.000 adultos deixaram de ser protegidos dos desfechos $\mathrm{CV}$ e foram internados por causas evitáveis e sensíveis a atenção básica relacionada à HAS (Tabela 4).

Em Bom Jardim da Serra, município com maior ineficiência na produção de resultados, o número de internações evitadas poderia ser aumentado em aproximadamente 66 adultos a cada 10.000. Considerando sua população adulta, 15 pessoas poderiam ter sido protegidas e não teriam sofrido internação por HAS, insuficiência cardíaca congestiva ou doenças cerebrovasculares. $\mathrm{O}$ valor observado nessa cidade foi de 17 internações, quando poderia ter reduzido o número de internações para duas para ser eficiente.

Dentre os 11 municípios eficientes na produção de resultados, nove $(81,9 \%)$ foram eficientes também na produção de serviços de atenção à HAS com impacto $(\mathrm{p}=0,001)$ (Tabela 5).

\section{DISCUSSÃO}

O modelo de avaliação desenvolvido no presente estudo mostrou-se capaz de medir a eficiência na atenção primária de saúde, ao avaliar a produtividade de serviços e de resultados.

A metodologia empregada pode ser ferramenta útil para os gestores municipais na reorientação atenção básica em busca da eficiência. Ela permite identificar tipos de recursos subutilizados, que aumento de serviços poderiam ocorrer com os recursos utilizados e o potencial de internações evitáveis por conseqüência da HAS.

A seleção de internações por causas sensíveis a atenção primária, ligadas aos cuidados da HAS e sua transformação em internações potencialmente evitáveis na população suscetível, resultou na criação da taxa de internações potencialmente evitadas, um marcador do impacto da atenção primária. Seu emprego mostrou-se útil para avaliar a eficiência de resultados.

O presente estudo avalia um nível de atenção com extenso espectro de atuação, porém detém-se ao conjunto de serviços relacionados ao controle da HAS, o que pode ser considerado como uma limitação. Camargo $\mathrm{Jr}$ et $\mathrm{al}^{4}$ afirmam que a avaliação pode ser restrita a certas condições ou patologias consideradas "representativas" das responsabilidades do sistema de assistência; portanto, vários estudos de sistemas, programas ou serviços de saúde utilizam esses "traçadores" como modo de avaliar o todo de um programa.

A assistência à HAS não é de exclusividade da APS, mas as melhores oportunidades de atuação acontecem no nível primário da atenção. A doença hipertensiva pode ser considerada um "traçador" da Saúde da Família, por ser um agravo priorizado na atenção à

Tabela 3. Municípios de referência para produção de resultados da atenção básica relacionados à hipertensão arterial sistêmica e seus insumos e produtos. Santa Catarina, 2007.

\begin{tabular}{lccccc}
\hline Município & $\begin{array}{c}\text { Vezes de } \\
\text { referência }\end{array}$ & $\begin{array}{c}\text { Vinculação do } \\
\text { usuário }\end{array}$ & $\begin{array}{c}\text { Atendimento } \\
\text { individual }\end{array}$ & $\begin{array}{c}\text { Pisita } \\
\text { domiciliar }\end{array}$ & $\begin{array}{c}\text { Taxa de internações } \\
\text { evitadas (por 10mil) }\end{array}$ \\
\hline Jaborá & 37 & 648 & 1.147 & 6.779 & $9.991,61$ \\
Jupiá & 32 & 224 & 1.834 & 2.675 & $9.991,57$ \\
Quilombo & 32 & 667 & 17.759 & 5.684 & $9.994,72$ \\
Belmonte & 12 & 211 & 3.833 & 4.093 & $9.991,68$ \\
Petrolândia & 11 & 362 & 570 & 10.103 & $9.988,04$ \\
Serra Alta & 10 & 491 & 547 & 4.737 & $9.988,21$ \\
Abdon Batista & 8 & 252 & 275 & 3.978 & $9.972,09$ \\
Cunhataí & 7 & 101 & 3.466 & 3.064 & $9.990,30$ \\
Santa Terezinha do Progresso & 5 & 241 & 958 & 4.845 & $9.981,38$ \\
Bandeirante & 1 & 280 & 225 & 4.988 & $9.961,09$ \\
Nova Itaberaba & 1 & 98 & 9.174 & 5.779 & $9.983,82$ \\
\hline
\end{tabular}


Tabela 4. Ineficiência na taxa de internações evitadas, meta de redução de desfechos cardiovasculares, desfechos observados e déficit na proteção aos desfechos relacionados ao controle da hipertensão arterial sistêmica na atenção básica. Santa Catarina, 2007.

\begin{tabular}{|c|c|c|c|c|c|}
\hline Município & $\begin{array}{c}\text { Ineficiência na } \\
\text { taxa de internações } \\
\text { evitadas por } 10 \text { mil }\end{array}$ & $\begin{array}{l}\text { População } \\
\text { adulta } \\
\text { suscetível }\end{array}$ & $\begin{array}{l}\text { Meta de redução de } \\
\text { desfechos CV em } \\
\text { número absoluto }\end{array}$ & $\begin{array}{c}\text { Desfecho } \\
\text { CV } \\
\text { observados }\end{array}$ & $\begin{array}{c}\text { Déficit na proteção } \\
\text { aos desfechos CV } \\
\text { em \% }\end{array}$ \\
\hline Bom Jardim da Serra & 65,9 & 2.257 & 14,86 & 17 & 87,4 \\
\hline Xavantina & 59,6 & 2.509 & 14,94 & 17 & 87,9 \\
\hline Alto Bela Vista & 47,6 & 1.067 & 5,07 & 6 & 84,6 \\
\hline Novo Horizonte & 43,3 & 1.546 & 6,69 & 8 & 83,7 \\
\hline Major Gercino & 42,8 & 1.537 & 6,57 & 8 & 82,1 \\
\hline Alfredo Wagner & 41,9 & 4.641 & 19,42 & 23 & 84,5 \\
\hline Bom Jesus do Oeste & 41,0 & 1.215 & 4,97 & 6 & 82,9 \\
\hline Lacerdópolis & 38,1 & 1.414 & 5,38 & 7 & 76,9 \\
\hline Tunápolis & 31,2 & 2.554 & 7,97 & 10 & 79,7 \\
\hline Santa Rosa de Lima & 31,2 & 1.252 & 3,90 & 5 & 78,1 \\
\hline São João do Oeste & 30,2 & 3.118 & 9,41 & 12 & 78,5 \\
\hline Treze de Maio & 27,8 & 4.227 & 11,73 & 16 & 73,3 \\
\hline Ermo & 26,7 & 1.237 & 3,30 & 6 & 55,0 \\
\hline Palma Sola & 26,0 & 4.125 & 10,71 & 14 & 76,5 \\
\hline Paial & 24,4 & 1.122 & 2,73 & 4 & 68,4 \\
\hline Arvoredo & 24,2 & 1.236 & 2,98 & 4 & 74,6 \\
\hline Vargem Bonita & 23,5 & 2.532 & 5,94 & 8 & 74,3 \\
\hline Bela Vista do Toldo & 23,1 & 3.258 & 7,51 & 10 & 75,1 \\
\hline Bom Retiro & 22,7 & 4.731 & 10,74 & 16 & 67,2 \\
\hline Vidal Ramos & 22,2 & 3.315 & 7,35 & 10 & 73,6 \\
\hline Planalto Alegre & 21,9 & 1.382 & 3,03 & 5 & 60,6 \\
\hline Timbó Grande & 20,3 & 3.870 & 7,85 & 11 & 71,4 \\
\hline Modelo & 19,2 & 2.174 & 4,18 & 6 & 69,7 \\
\hline São Pedro de Alcântara & 18,5 & 2.293 & 4,24 & 6 & 70,7 \\
\hline Irati & 18,5 & 1.090 & 2,01 & 3 & 67,0 \\
\hline Leoberto Leal & 18,0 & 1.897 & 3,40 & 5 & 68,1 \\
\hline Tigrinhos & 17,8 & 1.150 & 2,04 & 3 & 68,2 \\
\hline Marema & 17,6 & 1.378 & 2,42 & 4 & 60,7 \\
\hline Trombudo Central & 17,5 & 3.507 & 6,12 & 9 & 68,0 \\
\hline Canelinha & 17,0 & 5.703 & 9,71 & 14 & 69,4 \\
\hline Matos Costa & 15,5 & 2.108 & 3,26 & 5 & 65,3 \\
\hline Iraceminha & 14,6 & 2.185 & 3,18 & 5 & 63,6 \\
\hline Zortéa & 13,6 & 1.734 & 2,36 & 4 & 59,0 \\
\hline Descanso & 13,3 & 4.762 & 6,33 & 10 & 63,3 \\
\hline Anchieta & 11,8 & 3.030 & 3,56 & 7 & 50,9 \\
\hline Rio dos Cedros & 10,1 & 5.645 & 5,72 & 10 & 57,2 \\
\hline Ipira & 8,0 & 3.151 & 2,51 & 5 & 50,3 \\
\hline São Bernardino & 7,1 & 1.378 & 0,97 & 4 & 24,5 \\
\hline Calmon & 7,0 & 1.964 & 1,38 & 3 & 46,0 \\
\hline Guatambú & 7,0 & 2.645 & 1,83 & 4 & 46,0 \\
\hline Paulo Lopes & 6,2 & 3.589 & 2,23 & 5 & 44,7 \\
\hline Luzerna & 5,7 & 3.593 & 2,05 & 5 & 41,1 \\
\hline Águas Mornas & 5,4 & 3.076 & 1,66 & 5 & 33,2 \\
\hline
\end{tabular}

Continua 
Tabela 4 continuação

\begin{tabular}{lccccc}
\hline Município & $\begin{array}{c}\text { Ineficiência na } \\
\text { taxa de internações } \\
\text { evitadas por 10 mil }\end{array}$ & $\begin{array}{c}\text { População } \\
\text { adulta } \\
\text { suscetível }\end{array}$ & $\begin{array}{c}\text { Meta de redução de } \\
\text { desfechos CV em } \\
\text { número absoluto }\end{array}$ & $\begin{array}{c}\text { Desfecho } \\
\text { CV } \\
\text { observados }\end{array}$ & $\begin{array}{c}\text { Déficit na proteção } \\
\text { aos desfechos CV } \\
\text { em \% }\end{array}$ \\
\hline Caibi & 4,2 & 3.219 & 1,33 & 4 & 33,5 \\
Treze Tílias & 4,0 & 3.253 & 1,29 & 4 & 32,4 \\
Imbuia & 3,3 & 2.975 & 0,99 & 5 & 19,8 \\
Santiago do Sul & 2,7 & 900 & 0,24 & 1 & 24,7 \\
Painel & 1,7 & 1.535 & 0,26 & 4 & 6,5 \\
Erval Velho & 1,6 & 2.397 & 0,38 & 4 & 6,5 \\
Antônio Carlos & 1,4 & 4.254 & 0,61 & 5 & 15,3 \\
Irani & 1,4 & 5.509 & 0,78 & 4 & 15,8 \\
Romelândia & 0,8 & 2.363 & 0,18 & 1 & 4,5 \\
Ibiam & 0,4 & 1.122 & 0,04 & 1 & 4,5 \\
Águas Frias & 0,3 & 1.159 & 0,03 & 3 & 3,5 \\
Itá & 0,1 & 4.219 & 0,05 & 387 & 2,0 \\
\hline Total & 145.102 & 250,60 & & 64,8 \\
\hline
\end{tabular}

CV: Cardiovasculares

saúde do adulto, e, apesar de ser uma doença específica, é também um agravo caracterizado pela necessidade do cuidado longitudinal, característico da APS. ${ }^{17}$

Ao avaliar a produtividade dessa ação, não foram encontrados insumos exclusivos para esse agravo. $\mathrm{O}$ município é considerado ineficiente para uma ação específica, e não para a atuação da ESF como um todo, visto que os insumos são partilhados para todas as ações executadas nesse nível de atenção.

Outra restrição do estudo diz respeito à utilização de dados secundários. A utilização de dados dos Sistemas de Informações em Saúde (SIS) deve ser feita com cautela e análise crítica, pois é conhecido o risco de sub-registro e a baixa qualidade dos dados.

Autores de estudos de linha de base da atenção básica discordam sobre o tema. Camargo Jr et $\mathrm{al}^{4}$, ao analisarem três sistemas de informação, concluíram que o Siab possui cobertura e confiabilidade adequadas e que esses dados devem ser utilizados para a valorização dos sistemas de informação e para estimular seu uso como instrumentos de monitorização e avaliação. Fachini, ${ }^{9}$ por outro lado, afirma que as informações fornecidas pelos SIS são fragmentadas, desatualizadas e de baixa qualidade. Para Cordeiro et al, ${ }^{7}$ afirma que os dados do Siab não são desvalorizados pelo enfermeiro da equipe, e este fato pode repercutir na qualidade de sua coleta, levando em conta uma vez que estes profissionais são os supervisores das Agentes Comunitárias de Saúde. ${ }^{7}$ Apesar de não ter alcançado excelência total na qualidade dos dados oficiais, a qualificação desses dados no Brasil tem avançado e sua utilização contribui para esse avanço.
Tabela 5. Associação entre eficiência na produção de serviço e na produção de impacto relacionados à hipertensão arterial sistêmica desempenhados pela Estratégia Saúde da Família em municípios catarinenses. Santa Catarina, 2007.

\begin{tabular}{lccccccc}
\hline \multirow{2}{*}{$\begin{array}{l}\text { Eficiência na } \\
\text { produção de }\end{array}$} & \multicolumn{2}{c}{ Eficiência na produção de impacto } \\
serviço & $\mathrm{n}$ & $\%$ & $\mathrm{n}$ & $\%$ & $\mathrm{n}$ & $\%$ \\
\hline Sim & 9 & 36,0 & 16 & 64,0 & 25 & 37,9 \\
Não & 2 & 4,9 & 39 & 95,1 & 41 & 62,1 \\
\hline Total & 11 & 16,7 & 55 & 83,3 & 66 & 100,0 \\
\hline$Z$
\end{tabular}

$Z=3,28(p=0,001)$

Não foi possível avaliar a eficiência de 37 municípios devido à subnotificação ou aos erros de medida registrados nos bancos secundários. A qualificação dos registros, principalmente no cadastramento dos pacientes no Sishiperdia, poderá aprimorar as avaliações dos cuidados com HAS na atenção básica.

As publicações sobre a medida da eficiência em saúde estão concentradas em análises de sistemas de saúde nacionais e serviços hospitalares. Poucos são os estudos na atenção primária.

Um estudo de revisão realizado em 2000 sobre estudos de fronteira de eficiência na atenção primária identificou que tais estudos surgiram após a década de 1990 e concentravam-se em poucos países: Inglaterra, Espanha e Estados Unidos. Os estudos que se dirigiam a APS eram bastante reduzidos se comparados aos estudos de eficiência no setor saúde. Foram encontradas 25 publicações sobre o tema, das quais 21 utilizaram DEA como método de medida da eficiência. Entre 12 estudos espanhóis, a maioria utilizou dados secundários para a investigação. ${ }^{14}$ 
Puig-Junoy ${ }^{14}$ (2000) critica os estudos de eficiência em APS que utilizam somente indicadores de serviços e não avaliam sua qualidade, evidenciada pelos indicadores de resultados. Segundo o autor, apenas dois trabalhos empregavam esse tipo de indicador na Espanha. ${ }^{14}$

No presente estudo, foi utilizado indicador de resultado, uma vez que a taxa de prevenção às internações por causas sensíveis à atenção primária indica a obtenção de resultados, a qualidade do atendimento prestado em consultas individualizadas e visitas domiciliares, além da vinculação do usuário à equipe de saúde.

No Brasil, são poucas as publicações sobre eficiência na atenção básica. Em estudo com indicadores de insumos, produtos e resultados em municípios cearenses, foi observada maior eficiência em serviços do que em resultados, considerando todas as atividades de atenção básica executadas, como indicadores de serviços, e exclusivamente a mortalidade infantil e internação infantil por diarréia, como indicadores de resultados. ${ }^{d}$ Disso poderia decorrer a diminuição do número de municípios eficientes na produção de resultados. Esse mesmo resultado foi percebido no presente estudo, embora tenham sido aqui consideradas apenas as ações relacionadas ao agravo específico como indicadores de serviços.

A produção mais eficiente de serviços, sem a conseqüente produção eficiente de resultados, pode estar relacionada à falta de qualidade dos serviços, o que também foi aventado por Trompieri Neto et al (2008). ${ }^{\mathrm{d}}$

Varela et $\mathrm{al}^{\mathrm{e}}$ mediram a eficiência de serviços da atenção básica de 599 municípios de São Paulo. Os autores utilizaram dados secundários e constataram mudança do escore de eficiência em serviços ao analisarem outras variáveis não-controláveis relacionadas às características populacionais, a escala dos estabelecimentos de saúde e o percentual de recursos próprios aplicados em saúde. Esses fatores não foram analisados no presente estudo, mas grande parte foi controlada pelas restrições de porte e cobertura integral pela ESF. Sabe-se que há fatores externos aos indicadores selecionados que influenciam na produtividade de serviços e na produtividade de resultados, ${ }^{\mathrm{e}}$ mas que são desconhecidos no presente estudo.

Avaliações sobre efetividade predominam sobre eficiência em pesquisas avaliativas nacionais e internacionais relacionadas aos programas de controle de doenças cardiovasculares. Esses programas foram avaliados na atenção primária nos Estados Unidos, Noruega,
Paquistão e Suécia. Apesar de aplicados de formas distintas, possuem em comum o objetivo de diminuir os fatores de risco cardiovascular, incluindo a HAS. ${ }^{10,11,13,18}$

No Brasil, estudos sobre o tema também se concentram na análise de efetividade. Observou-se 98\% de adesão e impacto do PSF de $57 \%$ de controle da pressão arterial, com média de 10,1, $\pm 3,9$ consultas por ano na Bahia. ${ }^{2}$ Em São Paulo, a efetividade em uma unidade básica de saúde (não-Programa de Saúde da Família) foi de $44 \%$, com duas a quatro consultas anuais. ${ }^{15} \mathrm{Um}$ dos benchmarks na produção de serviços apresentou 1,6 consulta individual por ano para seus pacientes cadastrados no Sishiperdia em Santa Catarina. Além das consultas individuais, foram efetuadas cerca de 27 visitas domiciliares ao ano. Petrolândia, SC, obteve eficiência nos resultados a partir desses serviços.

Taxas de internação por Condições Sensíveis a Atenção Primária são usadas como indicador de qualidade e capacidade de resolução da atenção primária em variados estudos. ${ }^{5,12}$ Dentre os municípios ineficientes, 387 internações foram observadas. Para alcançar a eficiência em resultados, seria necessário que 250,6 internações potencialmente evitáveis não ocorressem, ou seja, $64,8 \%$ poderiam ter sido prevenidas nestes municípios, caso estes adotassem as práticas dos benchmarks.

Bermúdez-Tamayoa et $\mathrm{al}^{3}$ (2004) correlacionaram as internações por CSAP em hospitais de Granada, Espanha, com tipo de atenção, distância do hospital e fatores sociodemográficos e encontraram diferenças nas taxas da hospitalização por CSAP associadas às características organizativas da atenção primária, ao tamanho da municipalidade e à distância do hospital. ${ }^{3}$ A ineficiência encontrada no presente estudo não esteve sujeita ao porte ou ao tipo de atenção, pois os municípios formavam um grupo homogêneo de mesmo porte e mesmo tipo de atenção primária (ESF). A distância do hospital não foi analisada. No estudo espanhol, ${ }^{4}$ essa variável esteve relacionada ao porte do município e foi fator influente nos grandes municípios, os quais foram excluídos no presente estudo. Os padrões de internação podem ser influenciados por outros fatores, como a cultura de uso de serviços pela população e as políticas de admissão nos hospitais e a própria qualidade dos serviços prestados, que também não foram avaliados.

A metodologia aplicada permite a avaliação de custos evitáveis, e é possível calcular o gasto público em internações potencialmente evitáveis nos municípios e estimar o gasto desperdiçado em Santa Catarina por falta de eficiência na atenção básica.

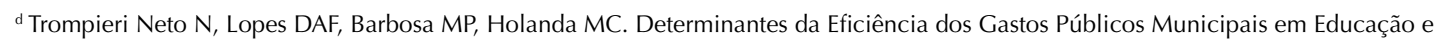
Saúde: O Caso do Ceará. IV Encontro Economia do Ceará em Debate. Fortaleza; 2008.

e Varela PS, Martins GA, Favero LPL. Desempenho e Accountability dos Municípios Paulistas: uma Avaliação de Eficiência na Atenção Básica à Saúde. Anais do III ANPCONT - International Accounting Congress. São Paulo; 2009.
} 


\section{REFERÊNCIAS}

1. Andrade LOM, Bueno ICHC, Bezerra RC. Atenção Primária à Saúde e Estratégia Saúde da Família. In: Campos GWS, Minayo MCS, Akerman M, organizadores. Tratado de Saúde Coletiva. Rio de Janeiro: Editora FIOCRUZ;2006. p.783-836.

2. Araujo JC, Guimaraes AC. Controle da hipertensão arterial em uma unidade de saúde da família. Rev Saude Publica. 2007;41(3):368-74. DOI:10.1590/ S0034-89102007000300007

3. Bermúdez-Tamayo C, Márquez-Calderón S, Rodriguez del Aguila MM, Perea-Milla López E, Ortiz Espinosa J. Caracteristicas organizativas de la atencion primaria y hospitalizacion por los principales ambulatory care sensitive conditions. Aten Primaria. 2004;33(6):305-11. DOI:10.1157/13059762

4. Camargo KR, Mascarenhas MTM, Mauad NM, Franco TB, David HMSL, Alves MJM, et al. Vivências e reflexões de avaliação na $A B$, a experiência dos ELBs/ Proesf em MG e ES. In: Hartz ZMA, Felisberto E, Silva LMV, organizadores. Meta-avaliação da atenção básica à saúde-teoria e prática. Rio de Janeiro: Editora FIOCRUZ 2008. p.119-52.

5. Caminal Homar J, Starfield B, Sánchez Ruiz E, Hermosilla Pérez E, Martín Mateo M. La atención primaria de salud y las hospitalizaciones por Ambulatory Care Sensitive Conditions en Cataluña. Rev Clin Esp. 2001;201(9):501-7.

6. Charnes A, Cooper WW, Rhodes E. Measuring the efficiency of decision making units. Eur J Opnl Res.1978;2(6):429-44. DOI:10.1016/03772217(78)90138-8

7. Cordeiro H, Pereira TR, Costa Jr H, Pereira ATS, Sulamis D. Acreditação como avaliação nos serviços de atenção primária em saúde. In: Hartz ZMA, Felisberto E, Silva LMV, organizadores. Meta-avaliação da atenção básica à saúde- teoria e prática. Rio de Janeiro: Editora FIOCRUZ;2008. p.153-66. [

8. Espigares JLN. Analisis de la eficiência em las organizaciones hospitalares públicas. Granada: Editora Universal de Granada; 1999. Análisis de la eficiencia en el entorno hospitalario: el análisis envolvente de datos; p.247-57.

9. Facchini LA, Piccini RX, Tomasi E, Thumé E, Silveira DS, Siqueira FV, et al. Desempenho do PSF no Sul e no Nordeste do Brasil: avaliação institucional e epidemiológica da atenção básica à saúde. Cienc
Saude Coletiva. 2006;11(3):669-81. DOI:10.1590/ S1413-81232006000300015

10. Lupton BS, Fønnebø V, Søgaard AJ, Langfeldt E. The Finnmark Intervention Study. Better health for the fishery population in an Arctic village in North Norway. Scand I Prim Health Care. 2002;20(4):213-8. DOI:10.1080/028134302321004863

11. Murray CJ, Lauer JA, Hutubessy RC, Niessen L, Tomijima N, Rodgers A, et al. Effectiveness and costs of interventions to lower systolic blood pressure and cholesterol: a global and regional analysis on reduction of cardiovascular-disease risk. Lancet. 2003;361(9359):717-25 DOI:10.1016/S01406736(03)12655-4

12. Nedel FB, Facchini LA, Martín-Mateo M, Vieira LAS, Thumé E. Programa Saúde da Família e condições sensíveis à atenção primária, Bagé (RS). Rev Saude Publica. 2008;42(6):1041-52. DOI:10.1590/S003489102008000600010

13. Nishtar S, Badar A, Kamal MU, Iqbal A, Bajwa R, Shah T, et al. The Heartfile Lodhran CVD prevention project--end of project evaluation. Promot Educ. 2007; 14(1):17-27.

14. Puig-Junoy J. Eficiencia en la atención primaria de salud: una revisión crítica de las medidas de frontera. Rev Esp Salud Publica. 2000;74(5-6):483-95. DOI:10.1590/S1135-57272000000500005

15. Sala A, Nemes Filho A, Eluf-Neto J. Avaliação da efetividade do controle da hipertensão arterial em unidade básica de saúde. Rev Saude Publica. 1996;30(2):161-7 DOI:10.1590/S003489101996000200008

16. Sancho LG. Eficiência. In: Sancho LG, editor. Avaliação econômica em saúde. São Paulo: Aderaldo \& Rothschild; 2007. p.61-77.

17. Starfield B. Avaliação da APS: uma visão da população. In: Starfield B, organizador. Atenção Primária: equilíbrio entre necessidade de saúde, serviços e tecnologia. Brasília: UNESCO, Ministério da Saúde; 2004. p.481-532.

18. Weinehall L, Lewis $C$, Nafziger AN, Jenkins PL, Erb TA, Pearson TA, et al. Different outcomes for different interventions with different focus!--A cross-country comparison of community interventions in rural Swedish and US populations. Scand I Public Health Suppl. 2001;56:46-58.

Trabalho baseado na dissertação de mestrado de Rabetti AC, apresentada à Universidade Federal de Santa Catarina em 2009.

Os autores declaram não haver conflitos de interesse. 